\title{
Residuos de fármacos anabolizantes en carnes destinadas al consumo humano
}

\author{
Álvaro L. Fajardo-Zapata, Francy J. Méndez-Casallas, Luis H. Molina \\ ${ }^{1}$ Grupo Salud Pública, Fundación Universitaria del Área Andina, Bogotá, D.C. Colombia. \\ -afajardo@areandina.edu.co; fmendez@areandina.edu.co
}

Recibido: 28-11-2010; Aceptado: 04-03-2011

\begin{abstract}
Resumen
La producción de carne para el consumo humano es uno de los renglones importantes dentro de la economía de un país, tiene una gran importancia socioeconómica ya que sirve como base para el desarrollo, generación de empleo y sostenibilidad. La demanda de proteína de origen animal es uno de los elementos más preciados y necesarios para mantener el adecuado desarrollo biológico de los seres humanos en crecimiento así como fuente de renovación de los tejidos. Este consumo ha aumentado porque las poblaciones y las migraciones hacia las ciudades crecen día tras día, lo cual genera que la oferta se torne insuficiente y se requiera acelerar, tecnificar y mejorar el proceso productivo de la carne. También se busca una disminución en los costos productivos por parte de los productores de este sector de la economía, lo que lleva a la utilización e incorporación de tecnología y de conocimientos de vanguardia con el fin de mejorar el rendimiento en la producción de carne; dentro de los nuevos conceptos se ha incorporado el conocimiento científico sobre crecimiento y desarrollo, trasladándolo al campo de la producción animal, lo que ha implicado el uso de hormonas y promotores del crecimiento (esteroides anabolizantes y otros), como fuentes para acelerar e incrementar la producción. Si bien es cierto, se ha logrado un incremento en la producción, los residuos de las sustancias usadas pueden quedar en la carne que se destina para el consumo humano y pueden generar alteraciones en el estado de salud de quienes la consumen.
\end{abstract}

Palabras clave: promotor, crecimiento, carne, producción animal.

\begin{abstract}
Residues of anabolic drugs in meat intended for human consumption. The production of meat for human consumption is one of the important items in the economy of a country. This activity has great socio economic importance as it serves as a basis for development, employment generation and sustainability of a country. The demand for animal protein is one of the most precious and necessary factors to maintain a proper biological development of human beings in active growth as well as being a source of growth and tissue regeneration. Consumption has increased because populations continue to grow and there is continuous migration to the cities, which makes the offer insufficient, and therefore it is necessary to accelerate, introduce technology and improve the meat production process. Industry also seeks a reduction in production costs by producers in this sector of the economy, leading to the use and incorporation of technology and cuttingedge knowledge to improve yield production of meat. Such knowledge comprises the scientific basis of the use of growth hormones and promoters (anabolic steroids and others) to accelerate and increase the production of meat. Although there has been an increase in production, residual substances can remain in the meat intended for human consumption which can lead to changes in the health of consumers.
\end{abstract}

Key words: promoter, growth, meat, animal production. 


\section{Resumo}

Resíduos de drogas anabolizantes em carnes para consumo humano. A produção de carne para consumo humano é um dos itens mais importantes na economia de um país, tem grande importância socioeconômica, uma vez que serve como base para o desenvolvimento, geração de emprego e sustentabilidade. A demanda por proteína animal é um dos elementos mais preciosos e necessários para manter o adequado desenvolvimento biológico dos seres humanos em crescimento; assim como, fonte de regeneração de tecidos. Este consumo tem aumentado porque as populações e as migrações para as cidades estão crescendo dia a dia, o que gera a oferta tornar-se insuficiente e seja necessário acelerar, tecnificar e melhorar o processo de produção de carne. Também se procura uma redução dos custos de produção pelos produtores desse setor da economia, levando à utilização e incorporação de tecnologia e de conhecimentos de vanguarda para melhorar o desempenho na produção de carne; dentro dos novos conceitos tem se incorporado o conhecimento científico sobre o crescimento e desenvolvimento, transferido para o campo da produção animal, que tem envolvido o uso de hormônios e promotores de crescimento (esteróides anabolizantes e outros), como fonte para acelerar e aumentar a produção. Embora, tenha havido um aumento na produção, os resíduos das substâncias usadas podem ficar na carne destinada ao consumo humano e podem levar a mudanças na saúde de quem consome.

Palavras-chave: promotor de crescimento, carne, produção animal.

\section{Introducción}

La meta principal de los productores de ganado destinados a la comercialización de carne para el consumo humano, ha sido siempre la de obtener mayores beneficios de sus productos, ya sea, utilizando razas precoces con mayor facilidad para el engorde o administrando sustancias, el mejoramiento de razas, la optimización de los sistemas de pastoreo, utilización de gramíneas y leguminosas, suplementación con subproductos, que al aumentar o modificar de alguna manera el metabolismo hagan que el animal transforme más el alimento que se le administra $(1,2)$.

Para lograr estos fines, los ganaderos pueden hacer uso de la producción limpia o mediante sustancias denominadas promotores de crecimiento, que abarcan desde el uso de hormonas sintetizadas por el mismo organismo para sus procesos biológicos, hasta sustancias sintéticas y que son administradas durante el desarrollo de los animales en fase de producción, a fin de acelerar dichos procesos y mejorar los rendimientos, bien sea disminuyendo el tiempo de crecimiento o aumentando la síntesis proteica $(1,2)$.

Si bien es cierto, se ha logrado un incremento en la producción, los residuos de las sustancias usadas pueden quedar en la carne que se destina para el consumo humano y pueden generar alteraciones en el estado de salud de quienes la consumen.

\section{Promotores del crecimiento}

En producción animal se define un promotor del crecimiento como todo aquel aditivo no esencial para la función biológica del animal, pero que tiene un efecto positivo como es el de mejorar el crecimiento y la eficiente conversión del alimento. Esto último significa que de una cantidad determinada de alimento, el metabolismo del animal puede obtener más energía y por consiguiente, producir más carne y menos cantidad de grasa (3).

Entre los promotores de crecimiento para alterar o modificar el metabolismo de los anímales se encuentran: uso de hormonas esteroideas, anabólicos sintéticos, hormona del crecimiento, agonistas beta adrenérgicos, antibióticos y el uso de alimentos transgénicos.

\section{Anabolizantes}

El uso de agentes anabólicos con actividad no hormonal es uno de los métodos no genéticos para modificar el potencial de crecimiento de los animales (4).

Numerosos países con sistemas intensivos de producción de carne utilizan anabólicos para mejorar su producción, especialmente la velocidad del crecimiento y conversión alimenticia. El objetivo de su utilización es acortar el período de producción y disminuir el insumo más caro: el tiempo (4).

Se define como anabólico esteroide cualquier compuesto o mezcla de compuestos que afectan la función metabólica del animal para incrementar la cantidad de proteína corporal. Los anabólicos pueden ser de origen endógeno (naturales) o sintéticos (4).

Entre los primeros se encuentran las hormonas naturales que incluyen el estradiol ( 17 beta y 17 alfa), la testosterona, la progesterona, la somatotrofina y los factores liberadores de esta última. En este mismo grupo se encuentran: los agonistas beta-adrenérgicos, como la epinefrina y norepinefrina, secretadas por la médula suprarrenal y las terminaciones nerviosas simpáticas. Su mecanismo de acción consiste en aumentar la ganancia de peso y la retención de nitrógeno (4). 
Los anabólicos esteroides sintéticos abarcan: el grupo de los estilbénicos (dietilestilbestrol y dienestrol) y los no estilbénicos (melengestrol, zeranol y trembolona) y los beta-adrenérgicos (clenbuterol, cimaterol y fenoterol) (4).

a. Estilbénicos: Están prohibidos en casi todo el mundo, y su componente más difundido es el dietilestilbestrol, conocido como DES. La prohibición se basa en que este producto, pese a ser barato y eficaz como engordador, tiene una alta acción estrogénica (feminizante).

b. No estilbénicos: Varios son los productos que contienen estas sustancias; los más conocidos son, dentro de los sintéticos, el zeranol, que es una hormona no natural, y la trembolona.

c. Agonistas beta-adrenérgicos de naturaleza sintética. Actúan incrementando la masa muscular, especialmente en animales de carne. Producen un cambio en el balance energético que cambia la relación carne-grasa. El clenbuterol fue el primer agonista sintético. Otros son el cimaterol y el fenoterol $(4,5)$.

La mayoría de los anabolizantes poseen propiedades fisiológicas similares a las de los esteroides sexuales naturales, con su uso, se pueden conseguir los siguientes efectos: aumento del ritmo de crecimiento, aumento de la masa muscular, mejoramiento de los índices de conversión, cambios en la distribución de la grasa corporal, mejoramiento del apetito y el aumento de la capacidad muscular para el trabajo.

Sin embargo, no todos estos efectos ocurren al mismo tiempo y su respuesta depende de varios factores como: especie, sexo, raza, edad, aptitud, etc. Así mismo también se debe considerar el tipo de sustancia que se utilice, la dosis que se administre y la vía de administración que se emplee.

\section{Administración de agentes anabólicos}

El tratamiento del ganado bovino con anabólicos hormonales naturales y sintéticos es un procedimiento que por décadas ha sido utilizado en países como Estados Unidos y por los beneficios económicos que ello representa para la industria pecuaria, ya que le permite obtener más kilogramos de carne en un menor tiempo y costo.

Entre las sustancias anabolizantes más ampliamente utilizadas se destacan el uso del acetato de trembolona con unos rendimientos significativos en cuanto a los índices de conversión y ganancia de peso se refiere (2).
En cuanto a las hormonas se destacan los antitiroideos, con singular importancia los derivados del tiuracilo, sustancias inhibidoras de la función tiroidea, cuya administración, debido a su interferencia en la síntesis de hormonas tiroideas, produce estados hipotiroideos mejoradores del incremento ponderal de peso vivo, en algunas especies zootécnicas (2).

Los agentes anabólicos pueden administrarse por vía oral o parenteral. Se suministran oralmente a los cerdos como aditivos del alimento al igual que en el caso de la cría intensiva de peces.

Los anabólicos se administran como implantes subcutáneos en bovinos, borregos y aves, o inyectados como soluciones oleosas en caballos y bovinos. El implante se coloca en la base de la oreja debido a que las hormonas que se administran son artificiales, y el organismo demora más en integrarlas. Se evita colocarlas en lugares que estén destinados para el consumo humano; una vez colocado el implante, la concentración de la hormona suplementada sube rápidamente y los residuos son mayores durante el período inicial después de la implantación. Por este motivo, cuando las hormonas son sintéticas, existe un tiempo denominado tiempo de retiro, el cual, debe transcurrir entre la fecha del implante y la fecha del sacrificio (véase tabla 5). El tiempo varía dependiendo del anabólico usado, y de la legislación de cada país. En el caso de hormonas naturales no es necesario que dicho período transcurra (3).

La implantación subcutánea en la base de la oreja, se realiza mediante una pistola de implante, en la cara dorsal de la oreja, a unos 2,5 cm de su inserción en la cabeza y evitando los bordes laterales del cartílago, lo cual permite una absorción lenta, debido a que el tejido implementado posee escasa irrigación sanguínea; asegurando de esta manera, un prolongado tiempo de acción que oscila entre los 90 y 400 días, dependiendo del anabólico empleado. Además, se afirma que la oreja es un tejido que se "desecha" (no se destina para el consumo humano) y de esta manera se asegura que los residuos que pudieran quedar en los tejidos adyacentes al sitio de implantación, sean normalmente descartados.

Se ha establecido que alrededor de 15 - 20\% de la dosis original permanecer sin absorberse en la base de la oreja.

Los sitios donde se realizan los implantes deberían desecharse al sacrificio, ya que es allí donde se encuentran los niveles hormonales más altos; sin embargo, se sabe con certeza que en Colombia tanto la oreja, como la papada, son tejidos consumidos por la población. 


\section{Administración de la hormona del crecimiento}

Un ejemplo clásico y más usado en el ganado bovino, es la hormona de crecimiento bovino, o somatotrofina, que es una hormona de tipo proteico constituida por 188 aminoácidos. Es secretada naturalmente por la hipófisis anterior bajo control del hipotálamo, en varios animales incluyendo la vaca.

Los efectos principales de esta hormona son: el incremento de la acumulación de proteínas (mayor retención de nitrógeno, el cual es un factor indispensable en la síntesis de proteínas), la disminución de la capa lipídica (la somatotrofina tiene varios efectos sobre las grasas), disminución de la glucogénesis, un aumento en la glucólisis y por último el aumento en la producción de leche (3).

\section{Hormonas en la producción avícola}

Desde el uso de los anabolizantes, uno de los efectos positivos se obtiene cuando se aplica en la etapa de crecimiento pospuberal. Tal es el caso de los pollos broilers que son procesados para el consumo en edad precoz, de 45 a 47 días de edad, lo que dista mucho de la etapa de pubertad, la cual se encuentra entre los 4 a 5 meses (3).

La aplicación de hormonas antes de la etapa puberal puede tener consecuencias negativas sobre el crecimiento, como la osificación de cartílagos y la consiguiente detención del crecimiento (3).

\section{Uso de antibióticos como promotores de crecimiento}

Mucho se ha hablado del uso de los antibióticos como promotores del crecimiento de animales destinados al consumo humano, del escaso control en su utilización y del riesgo sanitario de dicho uso. Hay que mencionar que las partes implicadas -ganaderos, industria farmacéutica y de producción de piensos, veterinarios, médicos y científicos en general-tienen opiniones diferentes respecto de la conveniencia o no del empleo de antibióticos como promotores de crecimiento (6).

La propiedad de los antibióticos de mejorar las tasas de crecimiento animal (aumentar la ganancia de peso y eficiencia de conversión) se conoce desde finales de los años cuarenta.

Los antibióticos como promotores de crecimiento se han empleado a dosis subterapéuticas durante largos períodos de la vida del animal, produciendo una ganancia de peso estimada alrededor del 5\%. El mecanismo por el cual los antibióticos favorecen el crecimiento no se conoce con exactitud. Básicamente actúan modificando cuantitativa y cualitativamente la flora microbiana intestinal, provocando una disminución de los microorganismos causantes de enfermedades subclínicas. Actúan también reduciendo la flora normal que compite con el huésped por los nutrientes. Todo ello conduce a una mejora en la productividad y reduce la mortalidad de los animales (6).

En 1970, la Comunidad Europea eliminó como promotores aquellos antibióticos que también fueran utilizados en la medicina humana o animal. De este modo, se prohibió en Europa el empleo de tetraciclinas o beta-lactámicos como promotores del crecimiento en el pienso de animales (en EE.UU. todavía se emplean estos antibióticos) (6).

En la actualidad en la comunidad europea, solamente quedan disponibles como promotores 4 antibióticos (avilamicina, flavofosfolipol, monensina sódica y salinomicina) y su utilización está siendo sometida a una reevaluación (6).

En EE.UU., actualmente están autorizadas como promotores del crecimiento la penicilina, clortetraciclina, eritromicina, estreptomicina, bacitracina y espectinomicina (todos estos antibióticos son de uso en humanos), tilosina y virginiamicina (antibióticos con estructuras similares a otros usados en humanos) (6).

Actualmente, ante el uso indiscriminado de antibióticos en la producción animal y con el fin de reducir su uso, se plantean nuevas alternativas como el uso de probióticos (microorganismos vivos que se agregan como suplemento a la dieta), prebióticos (ingredientes no digeribles de la dieta, que estimulan el crecimiento bacteriano en el colon) y simbióticos (son formulaciones que combinan probióticos con prebióticos que actúan sinérgicamente) los cuales representan una alternativa significativa y segura en la producción animal (7).

\section{Efectos potenciales de los residuos de fármacos en salud humana}

\section{Fármacos anabolizantes}

El uso de medicamentos veterinarios es esencial durante la crianza de animales con destino para el consumo humano. Todos los productos utilizados en la industria de la carne son utilizados con fines terapéuticos y preventivos en caso de infecciones o enfermedades y como se ha manifestado, se aplican como promotores del crecimiento (8). 
Las hormonas, como todas las sustancias medicamentosas y químicas, dejan en general residuos en las carnes que pueden ser detectados por sofisticados métodos aún en partes muy pequeñas. Sin embargo, existen dudas sobre si estos residuos pueden significar un riesgo para el consumidor (4).

Una cantidad apreciable de alimentos corrientes poseen normalmente hormonas sexuales en cantidades a veces no despreciables. Actualmente no existen investigaciones con resultados fidedignos que se refieran a la peligrosidad del uso de hormonas en la producción ganadera (3).

El Comité de Expertos sobre aditivos alimentarios de la Organización de Alimentos y Agricultura (FAO) y de la Organización Mundial de la Salud (OMS), la Administración de Drogas y Medicamentos (FDA) de los Estados Unidos de Norteamérica consideraron en 1988 que los residuos presentes en la carne de animales tratados con hormonas sexuales no representan riesgo alguno para el consumo humano (9).

La relación entre el consumo de carne y sus posibles consecuencias perjudiciales se basa, en el efecto de las hormonas y sustancias que actúan en el organismo de las mujeres, sobre el que existe un creciente número de indicios. Sin embargo, los autores de los estudios reconocen que no son concluyentes y que no se pueden descartar en los resultados la influencia de otros factores (10).

Estudios in vivo han demostrado rompimiento del ADN y daños oxidativos desencadenados por el 17- $\beta$ estradiol, por lo cual se considera que esta hormona tiene efecto genotóxico desencadenando, por ejemplo, la proliferación de células cancerígenas mamarias. Sin embargo, las dosis para que estas alteraciones ocurran son superiores a las que generan efectos endocrinos en los animales. Los efectos adversos de la testosterona, por su parte, se deben a su actividad hormonal, particularmente en la glándula prostática. Adicionalmente, la testosterona se considera potencialmente embriotóxica (10).

La utilización de somatotropina recombinante bovina ha generado controversia en los últimos años. Sus posibles efectos adversos destacan la posibilidad de desarrollar cáncer y diabetes, ambos efectos relacionados con el IGF-1 que aumenta en los tejidos animales por acción de la somatotropina (11).

El clembuterol es el principal fármaco sobre el cual se han reportado reacciones adversas en humanos por consumo de carne contaminada. Además de ser usado como anabolizante, el clembuterol es empleado como tocolítico (inhibidor de la contracción uterina) en hembras bovinas lo que supone un riesgo adicional. En España y Francia se reportó que el consumo de hígado de ternera con residuos de clenbuterol generó trémores musculares, taquicardia, dolor muscular, nerviosismo, dolor de cabeza, vértigo, náuseas, vómito y fiebre $(12,13)$.

En noviembre de 2005 en Jalisco, México, alrededor de 225 personas experimentaron temblor, dolor de cabeza y malestar después de haber consumido carne de res que contenía residuos de clenbuterol (14).

El dietilestilbestrol (DES) fue administrado mujeres embarazadas antes de los años 70 para prevenir abortos espontáneos. En 1971 se catalogó como un cancerígeno humano (cáncer de vagina y de cuello de útero). También se encontró que aumentaba la probabilidad de cáncer de mama (15).

En 1979, Herbst AL, Scully RE, Robboy SJ, hacen los primeros reportes de la asociación que existe entre la exposición prenatal a dietiletilbestrol y la presencia de cáncer de cérvix y vagina en niñas y adolescentes (16).

Herbs Al (1979) y Gerbie MV (1981), informaron sobre la aparición de carcinoma en niñas y adolescentes sometidas a dietilestilbestrol durante su vida fetal $(17,18)$.

La OMS, en su boletín farmacéutico 4 de 2004, reportó la relación entre el dietilestilbestrol y la adenocarcinoma, así como otras reacciones adversas en casos presentados desde 1971 y reportados por el boletín australiano de reacciones adversas a medicamentos en el volumen 23 , No. 3 de junio de 2004 (19).

\section{Fármacos antiinfecciosos}

El uso incorrecto de antibióticos es un factor que puede generar el desarrollo de resistencias bacterianas en los animales tratados. Estas bacterias resistentes podrían transmitirse al hombre causando dificultades en el momento de tratar infecciones humanas. Así mismo, los antibióticos consumidos por seres humanos provenientes de residuos presentes en alimentos de origen animal, generan una alteración de la flora intestinal y como consecuencia una disminución de bacterias que compiten con microorganismos patógenos, aumentando así el riesgo de enfermedad (14).

Varios antibióticos han sido reportados y sus residuos en alimentos pueden desencadenar reacciones alérgicas, causar hipersensibilidad, o ambas situaciones, entre ellos 
la penicilina, las sulfonamidas y la estreptomicina (20, 21).

Los nitrofuranos, empleados en el tratamiento de infecciones gastrointestinales en bovinos y porcinos, han sido prohibidos como medicamentos de uso veterinario en producción animal debido a los efectos carcinogénicos y mutagénicos ocasionados por sus metabolitos (22).

\section{CODEX Alimentarius}

El Codex Alimentarius es el organismo internacional que se ocupa de la ejecución del Programa Conjunto FAO/OMS sobre Normas Alimentarias, el cual tiene por objeto proteger la salud de los consumidores y asegurar prácticas equitativas en el comercio de los alimentos (Tabla 1).

En Colombia es muy poco lo que se conoce del Codex Alimentarius y la labor que cumple a nivel internacional; incluso, en el propio sector de alimentos del país, como empresas productoras, entidades oficiales, instituciones académicas o asociaciones científicas y tecnológicas, el desconocimiento de sus actividades es muy grande, no obstante el trabajo desarrollado por la Comisión del Codex Alimentarius es reconocido en el mundo entero por su invaluable aporte a la protección del consumidor y al comercio internacional.

Tabla 1. Medicamentos recomendados de uso veterinario referenciados por el Codex Alimentarius (23)

\begin{tabular}{|c|c|}
\hline Medicamento & Especie \\
\hline \multicolumn{2}{|r|}{ Antimicrobianos } \\
\hline Avilamicina & Cerdo, conejo, pollo, gallina, pavo. \\
\hline Bencilpenicilina/Bencilpenicilina procaínica & Toros, vacas, terneros, cerdo, pollo, gallina \\
\hline Ceptiofur & Toros, vacas, terneros, cerdo. \\
\hline Clortetraciclina/Oxitetraciclina/Tetraciclina & Toros, vacas, terneros, cerdo, oveja, aves, pescados, langostinos. \\
\hline Colistin & Toros, vacas, terneros, cerdo, oveja, cabra, conejo, pollo, gallina, pavo. \\
\hline Danofloxacina & Toros, vacas, terneros, cerdo, pollos, gallinas. \\
\hline Dihidroestreptomicina/Estreptomicina & Toros, vacas, terneros, cerdo, oveja, pollos, gallinas. \\
\hline Eritromicina & Pollos, gallinas, pavos. \\
\hline Espectinomicina & Toros, vacas, terneros, cerdo, pollos, gallinas. \\
\hline Espiramicina & Toros, vacas, terneros, cerdos, pollos, gallinas. \\
\hline Flumequina & Toros, vacas, terneros, cerdos, pollos, gallinas, ovejas, truchas. \\
\hline Gentamicina & Toros, vacas, terneros, cerdos. \\
\hline Lincomicina & Toros, vacas, terneros, cerdo, pollos, gallinas. \\
\hline Monensina & Toros, vacas, terneros, oveja, cabra, codorniz, pollos, gallinas, pavos. \\
\hline Narasina & Pollos, gallinas. \\
\hline Neomicina & Toros, vacas, terneros, cerdos, ovejas, cabras, pollos, gallinas, pavos, patos. \\
\hline Pirlimycina & Toros, vacas, terneros. \\
\hline Sarafloxacin & Pollos, gallinas, pavos. \\
\hline Sulfadimidina & Toros, vacas, terneros, no especificado. \\
\hline Tilmicosin & Toros, vacas, terneros, cerdos, ovejas. \\
\hline Tilosina & Toros, vacas, terneros, cerdos, ovejas, pollos, gallinas. \\
\hline
\end{tabular}

\begin{tabular}{ll}
\hline & \multicolumn{1}{c}{ Antiprotozoicos } \\
\hline Diclazuril & Oveja, conejos, aves. \\
Imidocarb & Toros, vacas, terneros. \\
Nicarbacina & Pollos, gallinas \\
\hline & Agonista adrenorreceptor \\
\hline Clenbuterol & Toros, vacas, terneros, caballos. \\
\hline
\end{tabular}




\section{Antihelmínticos}

Abamectin

Albendazol

Closantel

Doramectin

Eprinomectín

Febantel/Fenbendazol/Oxfendazol

Flubendazol

Ivermectina

Levamisol

Moxidectin

Tiabendazol

Triclabendazol
Toros, vacas, terneros.

No especificada

Toros, vacas, terneros, ovejas

Toros, vacas, terneros, cerdos.

Toros, vacas, terneros

Toros, vacas, terneros, cerdo, oveja, cabra, caballo

Cerdos, aves

Toros, vacas, terneros, cerdo, oveja.

Toros, vacas, terneros, cerdo, oveja, aves.

Toros, vacas, terneros, ovejas, ciervos.

Toros, vacas, terneros, cerdo, ovejas, cabras

Toros, vacas, terneros, ovejas.

\section{Bloqueante receptor adrenérgico beta}

Carazolol

Cerdos

\section{Coadyuvante de producción}

Acetato de melengestrol

Estradiol-17beta

Progesterona

Somatotropina porcina

Testosterona

Toros, vacas, terneros.

Toros, vacas, terneros.

Toros, vacas, terneros.

Cerdos.

Toros, vacas, terneros.

\section{Glucocorticosteroides}

Dexametasona

Toros, vacas, terneros, cerdos, caballos.

\section{Insecticidas}

Ciflutrín

Cihalotrin

Cipermetrina y alpfa-cypermetrina

Deltametrin

Diciclanil

Fluazuron

Foxim

Triclorfon (metrifonato)
Toros, vacas, terneros.

Toros, vacas, terneros, cerdos, ovejas.

Toros, vacas, terneros, ovejas.

Toros, vacas, terneros, ovejas, pollos, gallinas, salmón.

Ovejas

Toros, vacas, terneros.

Cerdos, ovejas, cabras.

Toros, vacas, terneros.

\section{Promotor del crecimiento}

Acetato de trembolona

Zeranol

Toros, vacas, terneros.

Toros, vacas, terneros.

\section{Tranquilizante}

Azaperona

Cerdos.

\section{Tripanosomicida}

Diminazina

Isometamidio
Toros, vacas, terneros.

Toros, vacas, terneros. 
Para las hormonas naturales, la FAO/OMS y el Codex Alimentarius reconocen que no tiene sentido establecer niveles mínimos de residuos, debido a que las cantidades de hormonas presentes en animales implantados son miles de veces menores que las cantidades generadas por las propias glándulas en el animal o en el hombre y la FDA (USA) no justifica establecer niveles mínimos de residuos cuando la ingesta es menor a 100 veces la producción de hormonas en el hombre (OMS, 1988), (Tabla 2) (11).

Tabla 2. Producción de hormonas humanas diarias (24)

\begin{tabular}{|c|c|c|c|}
\hline \multirow{2}{*}{$\begin{array}{l}\text { Hormona } \\
\begin{array}{l}\text { Estradiol } \\
\text { (suero) }\end{array}\end{array}$} & \multicolumn{2}{|c|}{ Estadio } & \multirow{2}{*}{$\begin{array}{l}\text { Unidades convencionales } \\
3 \text { a } 10 \mathrm{pg} / \mathrm{ml} \\
3 \text { a } 10 \mathrm{pg} / \mathrm{ml} \\
5 \text { a } 10 \mathrm{pg} / \mathrm{ml} \\
5 \text { a } 30 \mathrm{pg} / \mathrm{ml} \\
5 \text { a } 45 \mathrm{pg} / \mathrm{ml} \\
10 \text { a } 50 \mathrm{pg} / \mathrm{ml}\end{array}$} \\
\hline & Varón & $\begin{array}{l}1 \text { a } 5 \text { años } \\
6 \text { a } 9 \text { años } \\
10 \text { a } 11 \text { años } \\
12 \text { a } 14 \text { años } \\
15 \text { a } 17 \text { años } \\
>\text { de } 17 \text { años }\end{array}$ & \\
\hline & Mujer & $\begin{array}{l}1 \text { a } 5 \text { años } \\
6 \text { a } 9 \text { años } \\
10 \text { a } 11 \text { años } \\
12 \text { a } 14 \text { años } \\
15 \text { a } 17 \text { años } \\
\text { Folicular temprano } \\
\text { Folicular tardío } \\
\text { Umbral de la mitad de ciclo } \\
\text { Luteínico } \\
\text { Posmenopáusica }\end{array}$ & $\begin{array}{l}5 \text { a } 10 \mathrm{pg} / \mathrm{ml} \\
5 \text { a } 60 \mathrm{pg} / \mathrm{ml} \\
5 \text { a } 300 \mathrm{pg} / \mathrm{ml} \\
25 \text { a } 410 \mathrm{pg} / \mathrm{ml} \\
40 \text { a } 410 \mathrm{pg} / \mathrm{ml} \\
20 \text { a } 150 \mathrm{pg} / \mathrm{ml} \\
40 \text { a } 350 \mathrm{pg} / \mathrm{ml} \\
150 \text { a } 750 \mathrm{pg} / \mathrm{ml} \\
30 \text { a } 450 \mathrm{pg} / \mathrm{ml} \\
<20 \mathrm{pg} / \mathrm{ml}\end{array}$ \\
\hline $\begin{array}{l}\text { 17- Hidroprogesterona } \\
\text { (suero) }\end{array}$ & & $\begin{array}{l}1 \text { a } 6 \text { meses } \\
6 \text { a12 meses } \\
\text { Niños prepúberes } \\
\text { Púberes } \\
\text { Adultos }\end{array}$ & $\begin{array}{l}36 \text { a } 763 \mathrm{ng} / \mathrm{dl} \\
42 \text { a } 540 \mathrm{ng} / \mathrm{dl} \\
15 \text { a } 221 \mathrm{ng} / \mathrm{dl} \\
44 \text { a } 235 \mathrm{ng} / \mathrm{dl} \\
53 \text { a } 357 \mathrm{ng} / \mathrm{dl}\end{array}$ \\
\hline & Varón & $\begin{array}{l}1 \text { a } 5 \text { años } \\
6 \text { a } 9 \text { años } \\
10 \text { a } 11 \text { años } \\
12 \text { a } 14 \text { años } \\
15 \text { a } 17 \text { años } \\
\text { Adulto }\end{array}$ & $\begin{array}{l}\text { Menor o igual a } 12 \mathrm{ng} / \mathrm{dl} \\
3 \text { a } 30 \mathrm{ng} / \mathrm{dl} \\
5 \text { a } 50 \mathrm{ng} / \mathrm{dl} \\
10 \text { a } 572 \mathrm{ng} / \mathrm{dl} \\
220 \text { a } 800 \mathrm{ng} / \mathrm{dl} \\
260 \text { a } 1000 \mathrm{ng} / \mathrm{dl}\end{array}$ \\
\hline & Mujeres & $\begin{array}{l}1 \text { a } 5 \text { años } \\
6 \text { a } 9 \text { años } \\
10 \text { a } 11 \text { años } \\
12 \text { a } 14 \text { años } \\
15 \text { a } 17 \text { años } \\
\text { Adulta premenopáusica }\end{array}$ & $\begin{array}{l}\text { Menor o igual a } 12 \mathrm{ng} / \mathrm{dl} \\
2 \text { a } 20 \mathrm{ng} / \mathrm{dl} \\
5 \text { a } 25 \mathrm{ng} / \mathrm{dl} \\
10 \text { a } 40 \mathrm{ng} / \mathrm{dl} \\
5 \text { a } 40 \mathrm{ng} / \mathrm{dl} \\
15 \text { a } 70 \mathrm{ng} / \mathrm{dl}\end{array}$ \\
\hline $\begin{array}{l}\text { Testosterona total } \\
\text { (suero) }\end{array}$ & & Adulta posmenopáusica & 5 a $51 \mathrm{ng} / \mathrm{dl}$ \\
\hline $\begin{array}{l}\text { Hormona del crecimiento } \\
\text { (suero) }\end{array}$ & & $\begin{array}{l}\text { Niños } \\
\text { Adultos }\end{array}$ & $\begin{array}{l}<13 \mathrm{ng} / \mathrm{ml} \\
<5 \mathrm{ng} / \mathrm{ml}\end{array}$ \\
\hline & Hombres & Adultos & $<1.4 \mathrm{ng} / \mathrm{ml}$ \\
\hline $\begin{array}{l}\text { Progesterona } \\
\text { (suero) }\end{array}$ & Mujeres & $\begin{array}{l}\text { Fase folicular } \\
\text { Fase luteal } \\
\text { Posmenopáusica } \\
\text { Embarazo primer trimestre } \\
\text { Embarazo segundo trimestre } \\
\text { Embarazo tercer trimestre }\end{array}$ & $\begin{array}{l}<1.4 \mathrm{ng} / \mathrm{ml} \\
3.3 \text { a } 26.0 \mathrm{ng} / \mathrm{L} \\
<0.7 \mathrm{ng} / \mathrm{ml} \\
11.0 \text { a } 45.0 \mathrm{ng} / \mathrm{L} \\
26.0 \text { a } 89.0 \mathrm{ng} / \mathrm{L} \\
46.0 \text { a } 423.0 \mathrm{ng} / \mathrm{L}\end{array}$ \\
\hline
\end{tabular}




\section{Control de residuos de fármacos}

El Codex Alimentarius junto con la FAO/OMS se ocupa de la ejecución del programa sobre normas alimentarias, dentro del cual se establecen los límites máximos de residuos en los alimentos que garanticen la protección de la salud de los consumidores. En la tabla 3 se muestran los límites máximos de residuos de promotores de crecimiento recomendados en el Programa.

\section{Legislación para los fármacos veterinarios comercializados en el mundo}

Directiva 2003/74/CE del Parlamento Europeo y del Consejo del 22 de septiembre de 2003 que modifica la Directiva 96/22/CE del Consejo por la que se prohíbe utilizar determinadas sustancias de efecto hormonal y tireostático y sustancias B-agonistas en la cría de ganado (25).

Tabla 3. Norma alimentaria de los límites máximos de residuos de promotores de crecimiento recomendados por el Codex alimentarius (23)

\begin{tabular}{|c|c|c|c|c|c|}
\hline Medicamento & Especie & Tejido & $\begin{array}{c}\text { LMR } \\
(\mu \mathrm{g} / \mathrm{kg})\end{array}$ & $\begin{array}{c}\text { Año } \\
\text { de adopción }\end{array}$ & Nota \\
\hline \multicolumn{6}{|c|}{ Agonista adrenorreceptor } \\
\hline Clenbuterol & $\begin{array}{c}\text { Vacuno/vaca } \\
\text { Vacuno/vaca } \\
\text { Vacuno/vaca } \\
\text { Vacuno/vaca } \\
\text { Vacuno/vaca } \\
\text { Caballo } \\
\text { Caballo } \\
\text { Caballo } \\
\text { Caballo }\end{array}$ & $\begin{array}{l}\text { Hígado } \\
\text { Músculo } \\
\text { Leche } \\
\text { Grasa } \\
\text { Riñón } \\
\text { Grasa } \\
\text { Músculo } \\
\text { Riñón } \\
\text { Hígado }\end{array}$ & $\begin{array}{c}0,6 \\
0,2 \\
0,05 \\
0,2 \\
0,6 \\
0,2 \\
0,2 \\
0,6 \\
0,6\end{array}$ & 2003 & $\begin{array}{l}\text { Debido a la posibilidad de abuso de } \\
\text { este medicamento, los LMR se reco- } \\
\text { miendan únicamente cuando estén } \\
\text { asociados con un uso terapéutico } \\
\text { aprobado a nivel nacional, por } \\
\text { ejemplo para tocólisis o como te- } \\
\text { rapia complementaria en enfermeda- } \\
\text { des respiratorias. }\end{array}$ \\
\hline \multicolumn{6}{|c|}{ Bloqueante receptor adrenérgico beta } \\
\hline Carazolol & $\begin{array}{l}\text { Cerdo } \\
\text { Cerdo } \\
\text { Cerdo } \\
\text { Cerdo }\end{array}$ & $\begin{array}{c}\text { Grasa/piel } \\
\text { Músculo } \\
\text { Hígado } \\
\text { Riñón }\end{array}$ & $\begin{array}{c}5 \\
5 \\
25 \\
25\end{array}$ & 1993 & $\begin{array}{l}\text { La concentración en el punto de in- } \\
\text { yección dos horas después del trata- } \\
\text { miento puede resultar en una ingestión } \\
\text { que exceda la DRF (dosis de referen- } \\
\text { cia) aguda y, por tanto, se debe aplicar } \\
\text { un período de retirada adecuado. }\end{array}$ \\
\hline
\end{tabular}

\begin{tabular}{|c|c|c|c|c|}
\hline \multicolumn{5}{|c|}{ Glucocorticosteroides } \\
\hline \multirow[t]{10}{*}{ Dexametasona } & Vacuno/vaca & Músculo & 1 & 2009 \\
\hline & Vacuno/vaca & Leche & 0,3 & \\
\hline & Vacuno/vaca & Hígado & 2 & \\
\hline & Vacuno/vaca & Riñón & 1 & \\
\hline & Cerdo & Riñón & 1 & \\
\hline & Cerdo & Hígado & 2 & \\
\hline & Cerdo & Músculo & 1 & \\
\hline & Caballo & Músculo & 1 & \\
\hline & Caballo & Riñón & 1 & \\
\hline & Caballo & Hígado & 2 & \\
\hline \multicolumn{5}{|c|}{ Promotor del crecimiento } \\
\hline \multirow{2}{*}{$\begin{array}{l}\text { Acetato de } \\
\text { trembolona }\end{array}$} & Vacuno/vaca & Hígado & 10 & 1995 \\
\hline & Vacuno/vaca & Músculo & 2 & \\
\hline \multirow[t]{2}{*}{ Zeranol } & Vacuno/vaca & Hígado & 10 & \\
\hline & Vacuno/vaca & Músculo & 2 & \\
\hline
\end{tabular}




\begin{tabular}{|c|c|c|c|c|c|}
\hline \multicolumn{6}{|c|}{ Coadyuvante de producción } \\
\hline $\begin{array}{l}\text { Acetato de } \\
\text { melengestrol }\end{array}$ & $\begin{array}{l}\text { Vacuno/vaca } \\
\text { Vacuno/vaca } \\
\text { Vacuno/vaca } \\
\text { Vacuno/vaca }\end{array}$ & $\begin{array}{l}\text { Grasa } \\
\text { Músculo } \\
\text { Hígado } \\
\text { Riñón }\end{array}$ & $\begin{array}{c}18 \\
1 \\
10 \\
2\end{array}$ & 2009 & \multirow{5}{*}{$\begin{array}{l}\text { Es improbable que los } \\
\text { residuos derivados del } \\
\text { uso de esta sustancia, } \\
\text { como estimuladora del } \\
\text { crecimiento de acuerdo } \\
\text { con las buenas prácticas } \\
\text { zootécnicas, represente un } \\
\text { peligro para la salud } \\
\text { humana. }\end{array}$} \\
\hline Estradiol-17 beta & $\begin{array}{l}\text { Vacuno/vaca } \\
\text { Vacuno/vaca } \\
\text { Vacuno/vaca } \\
\text { Vacuno/vaca }\end{array}$ & $\begin{array}{c}\text { Grasa } \\
\text { Hígado } \\
\text { Riñón } \\
\text { Músculo }\end{array}$ & $\begin{array}{l}\text { innecesario } \\
\text { innecesario } \\
\text { innecesario } \\
\text { innecesario }\end{array}$ & $\begin{array}{l}1995 \\
2005\end{array}$ & \\
\hline Progesterona & $\begin{array}{l}\text { Vacuno/vaca } \\
\text { Vacuno/vaca } \\
\text { Vacuno/vaca } \\
\text { Vacuno/vaca }\end{array}$ & $\begin{array}{c}\text { Grasa } \\
\text { Hígado } \\
\text { Riñón } \\
\text { Músculo }\end{array}$ & $\begin{array}{l}\text { Innecesario } \\
\text { Innecesario } \\
\text { Innecesario } \\
\text { Innecesario }\end{array}$ & 2003 & \\
\hline $\begin{array}{l}\text { Somatotropina } \\
\text { porcina }\end{array}$ & $\begin{array}{l}\text { Cerdo } \\
\text { Cerdo } \\
\text { Cerdo } \\
\text { Cerdo } \\
\end{array}$ & $\begin{array}{c}\text { Grasa } \\
\text { Hígado } \\
\text { Riñón } \\
\text { Músculo } \\
\end{array}$ & $\begin{array}{l}\text { No especificado } \\
\text { No especificado } \\
\text { No especificado } \\
\text { No especificado }\end{array}$ & & \\
\hline Testosterona & $\begin{array}{l}\text { Vacuno/vaca } \\
\text { Vacuno/vaca } \\
\text { Vacuno/vaca } \\
\text { Vacuno/vaca }\end{array}$ & $\begin{array}{l}\text { Riñón } \\
\text { Grasa } \\
\text { Hígado } \\
\text { Músculo }\end{array}$ & $\begin{array}{l}\text { Innecesario } \\
\text { Innecesario } \\
\text { Innecesario } \\
\text { Innecesario }\end{array}$ & 1995 & \\
\hline
\end{tabular}

La Directiva 96/22/CE del Consejo establece que los estados miembros deben prohibir la administración de sustancias que tengan, entre otras cosas, un efecto estrogénico, androgénico o gestágeno a los animales de explotación (el estradiol-17 $\mathrm{B}$, la testosterona, la progesterona, el acetato de trembolona, el zeranol y el acetato de melengestrol). La administración de estas sustancias a los citados animales está autorizada exclusivamente con fines terapéuticos o para un tratamiento zootécnico de acuerdo con las disposiciones de los artículos 4, 5 y 7 de dicha directiva (26).

En el caso particular de la utilización del estradiol-17 ß, existen pruebas de peso que apuntan a que esta sustancia se debe considerar carcinógeno completo, ya que provoca tumores y favorece su desarrollo, y los datos actualmente disponibles no permiten hacer una estimación cuantitativa del riesgo. El comité de medicamentos veterinarios de la Unión Europea observó, en particular, que el estradiol-17 ß únicamente ejerce su acción carcinógena tras una exposición prolongada y a niveles considerablemente superiores a los exigidos para una respuesta fisiológica (estrogénica) (26).

En el caso concreto de: la testosterona, la progesterona, el acetato de trembolona, el zeranol y el acetato de melengestrol), el comité científico considera que, a pesar de todos los datos toxicológicos y epidemiológicos disponibles que se han tenido en cuenta, el actual estado de conocimientos no permite realizar una estimación cuantitativa del riesgo para los consumidores (26).
La prohibición provisional de estas cinco hormonas debe aplicarse mientras la Comunidad Europea busque datos científicos más completos, cualquiera que sea su origen, que puedan arrojar luz y aclarar las deficiencias del actual estado de conocimientos sobre estas sustancias (26).

El empleo veterinario terapéutico de beta-agonistas sólo ha sido aprobado en el caso del clenbuterol como broncodilatador en caballos y terneros y como tocolítico en vacas. No se permite el uso de ningún beta-agonista en la Comunidad Europea para objetivos que promuevan el crecimiento en animales de granja (26).

La Organización Mundial de la Salud en su Boletín Farmacéutico No. 4 de 2004, reportó la relación entre el dietilestilbestrol y la adenocarcinoma, así como otras reacciones adversas en casos presentados desde 1971 y reportados por el Boletín Australiano de Reacciones Adversas a Medicamentos en el volumen 23, No. 3 de junio de 2004 (19).

En Estados Unidos, está permitido el uso de los anabólicos en la producción animal, con excepción de los agonistas. La FDA autoriza los implantes de Zeranol en terneros a partir del momento del nacimiento, pero nunca debe aplicarse, por motivos higiénico-sanitarios, en los 65 días previos al sacrificio. Para la asociación de estrógenos, progesteronas y andrógenos está permitido obviarse el período de supresión del tratamiento previo al sacrificio. La FDA no exige el período de supresión del implante de 17 Beta-estradiol antes del sacrificio. 
Argentina: prohíbe el uso de hormonas y sus normas controlan los residuos en las carnes por medio de metodologías que se basan en técnicas de cromatografía y de radioinmunoanálisis. El undecilato de boldenona está prohibido en: Uruguay, Venezuela, Brasil y Paraguay.

Australia apoya la posición de los norteamericanos; Brasil y Uruguay no tienen permitido el uso del zeranol, boldenona, pero sí admiten el empleo de otras sustancias anabólicas. En Paraguay están prohibidos los anabolizantes destinados a los animales de consumo. El dietilestilbestrol, está prohibido en el mundo excepto en Colombia.

Legislación para los fármacos veterinarios comercializados en Colombia

Desde 1998 Colombia crea oficialmente el Comité Nacional del Codex Alimentarius para formular políticas relacionadas con los procesos adelantados en la Comisión mixta FAO/ OMS de este organismo, en la que se incluye la regulación de residuos de medicamentos en productos animales (27).

En 2001 el Instituto Colombiano Agropecuario (ICA), como entidad responsable de la calidad de los alimentos de origen pecuario producidos en Colombia, creó el "Grupo de inocuidad de las cadenas agroalimentarias pecuarias", que junto con el Laboratorio Nacional de
Insumos Pecuarios, también dependencia del ICA, tienen la responsabilidad de adelantar acciones destinadas a fomentar la inocuidad de los alimentos a través del control de residuos de fármacos utilizados en animales destinados para el consumo humano.

En la actualidad en Colombia se comercializan varios tipos de esteroides anabólicos inyectables, los que poseen la licencia del ICA se relacionan en la tabla 5 .

\section{Técnicas para la detección de residuos}

La presencia de hormonas esteroideas o sus metabolitos en animales destinados para el consumo humano ha sido el sujeto de investigación durante los últimos 40 años.

Las técnicas para la detección de residuos más utilizadas son la espectrometría de masas y/ la cromatografía líquida; siendo la primera, la técnica de elección (33).

Chrusch, en Canadá, Xu Cl, en China, Blasco, en España, y Giannetti en Italia, estudiaron la cromatografía líquida y la espectrometría de masas como métodos efectivos en la determinación de por lo menos 29 residuos en tejidos de animales de sustancias o medicamentos administrados. Estos métodos son reconocidos como precisos y selectivos (34-36).

Tabla 4. Resoluciones y observaciones para fármacos veterinarios comercializados en Colombia

\section{Resolución Observaciones}

Resolución ICA 991 de 2004.

Por la cual se prohíbe el uso y comercialización del Dimetridazol (desparasitante) para uso animal (28).

Resolución ICA 1966 de 1984

Por el cual se reglamenta el uso de productos o sustancias antimicrobianas como promotores de crecimiento o mejorados de la eficiencia alimenticia (29).

Resolución ICA 1082 de 1995.

Por la cual se prohíbe el uso y comercialización de la Furazolidona, la Nitrofurazona y la Furaltadona para uso animal (31).

Resolución ICA 1326 de 1981

Por la cual se adoptan las disposiciones para la utilización y comercialización de productos antimicrobianos de uso veterinario (32).

Proyecto de resolución

Por medio de la cual se prohíbe la importación, comercialización y uso de dietilestilbestrol en la producción de insumos veterinarios, así como su administración en cualquier especie animal. Actualmente el uso del dietilestilbestrol está permitido en Colombia.

Fuente: autores. 
Tabla 5. Productos de producción y comercialización con licencia por el ICA para ser usados en Colombia y de uso restringido

\begin{tabular}{|c|c|c|c|}
\hline Producto & Tipo de producto & Uso & $\begin{array}{c}\text { Tiempo en días } \\
\text { de retiro antes } \\
\text { del sacrificio } \\
\text { del animal }\end{array}$ \\
\hline Zeranol & Estrógeno(no hormonal) & $\begin{array}{l}\text { es un anabólico no hormonal, que promueve el } \\
\text { crecimiento y engorde, logrando mayor ganancia de kilos } \\
\text { en menos tiempo, al aumentar la fijación del nitrógeno y } \\
\text { su transformación en proteínas }\end{array}$ & 28 \\
\hline Boldenona undecilato & Esteroide anabólico & $\begin{array}{l}\text { Derivado de la testosterona con efecto anabolizante. } \\
\text { Coadyuvante en casos que se requiera promover la } \\
\text { síntesis proteica en enfermedades de tipo consuntivo o } \\
\text { debilitantes que produzcan pérdida de peso, raquitismo } \\
\text { o fracturas. Es una sustancia que favorece el crecimiento } \\
\text { de los tejidos. }\end{array}$ & 30 \\
\hline Acetato de trembolona & Esteroide & $\begin{array}{l}\text { Este esteroide se usa para obtener una carne más magra } \\
\text { en los animales. }\end{array}$ & 65 \\
\hline Clenbuterol & $\begin{array}{l}\text { Agonista de los receptores } \\
\text { beta-2 }\end{array}$ & $\begin{array}{l}\text { Es usado en muchos países como un bronco dilatador. } \\
\text { Se usa para engorde artificial de ganado. }\end{array}$ & 65 \\
\hline 17 beta estradiol & Estrógeno natural & $\begin{array}{l}\text { Se usa para impedir la formación de quistes ováricos, } \\
\text { sincronizador de ovulación, o inductor de celo, retención } \\
\text { placentaria, endometriosis en vacas }\end{array}$ & 14 \\
\hline Dietilestilbestrol (DES) & Estrógeno sintético & Anabólico sintético & 15 \\
\hline Taleranol & Anabólico & Anabólico de la familia del zeranol. & - \\
\hline Dexametasona & Glucocorticoide & Su acción es antiinflamatoria & 15 \\
\hline Progesterona & Hormona esteroidea & Hormona esteroidea implicada en el ciclo menstrual & 21 \\
\hline Somatotropina & Hormona del crecimiento & Induce el crecimiento de los tejidos. & - \\
\hline Testosterona & Hormona androgénica & $\begin{array}{l}\text { Se usa para aumentar la masa muscular y el volumen en } \\
\text { los animales }\end{array}$ & 120 \\
\hline
\end{tabular}

Fuente: Instituto Colombiano Agropecuario (ICA) 2010.

En Francia, Destrez y otros han estudiado la presencia de la boldenona (prohibida en la comunidad europea) y sus metabolitos en orina de animales mediante cromatografía líquida y espectrometría de masas en animales de carne para consumo humano. Las conclusiones son que estos métodos son satisfactorios para las determinaciones de mínimas partes de las sustancias prohibidas y que pudieran ser usadas ilegalmente como promotores de crecimiento (37).

En Japón, Furusawa, determinó que la cromatografía líquida (HPLC) es un método fácil, barato, inofensivo y rápido para el análisis de acetato de trembolona y 17 betatrembolona en músculo de ganado (38).

La detección de residuos de esteroides en el pelo es una estrategia poderosa de demostrar la administración a largo plazo de estos promotores de crecimiento en animales de producción de carne. El análisis de la forma de éster de esteroides administrados es un acercamiento inequívoco de demostrar el empleo ilegal de hormonas naturales. Para la detección, la espectrometría de masas generalmente era usada. Sin embargo, avances recientes en la espectrometría de masa cromatografía líquida (LC-MS/MS) han mejorado la robustez y la sensibilidad potencial de este método tal y como lo demostraron Duffy y otros en Irlanda del Norte (39).

La detección de abuso de glucocorticoides (dexametasona) en la orina bovina, por HPLC, fue investigado por Vincenti et al., en Italia y Connolly en Irlanda del Norte. Estos autores y sus grupos concluyeron que la HPLC es un buen método para la detección del uso ilegal de este glucocorticoide $(40,41)$. 
En Italia, Vascellari y su grupo estudiaron el estado histológico del timo, y la concentración de cortisol en sangre como biomarcadores indirectos para detectar el empleo ilegal de corticosteroides en la producción de carne. La metodología para el análisis del timo tuvo una sensibilidad del 100 por ciento y una especificidad del 85 por ciento (42).

Sawaya, en Kuwait, estudió la presencia de cualquier residuo de promotor de crecimiento en muestras de orina de oveja, en carne de pollo y de ternera, usando la técnica de ELISA para determinar la precisión y exactitud de esta metodología. Los autores compararon esta metodología, contra la HPLC, y espectrometría de masas y concluyeron que la técnica de ELISA es una muy buena metodología que puede ser usada en la determinación de residuos de promotores de crecimiento (43).

Salim Mattar y colaboradores, en Colombia, determinaron la presencia de penicilina, oxitetraciclina y cloranfenicol, y encontraron estas sustancias en el $25 \%$ de las leches muestreadas (44).

Franco Jackeline y otros en Colombia, determinaron los niveles residuales de tetraciclina en canales bovinos por la técnica de Elisa, y encontraron una proporción de muestras de carne con concentraciones de antibióticos superiores a los LMR utilizados como punto de referencia (45).

Marín Lasso, y colaboradores en Colombia, estudiaron los niveles sanguíneos de ampicilina en pollos de engorde (de 49 días de edad) (46).

\section{Conclusiones}

La producción de animales cuya carne es destinada para el consumo humano es un renglón muy importante de la economía, y factor fundamental en el desarrollo de los seres humanos, porque no se debe desconocer la vital importancia de las proteínas de origen animal en los procesos biológicos humanos. Si bien la carne aporta proteínas fundamentales, su ingesta puede representar en algunos casos el consumo de sustancias extrañas al organismo, las cuales pudieron ser usadas para acelerar los procesos metabólicos de crecimiento de los animales y que pueden generar toxicidad aguda o crónica en quienes las consumen.

En la actualidad, se sabe que la industria de producción animal utiliza productos químicos denominados promotores de crecimiento que como se manifestó, buscan acelerar los procesos de crecimiento y aumentar la producción de carne. Sin embargo, el impacto de estas sustancias extrañas al cuerpo humano, incluyendo su farmacocinética y efectos a nivel molecular, requieren de investigación exhaustiva antes de establecer de manera definitiva su aparente inocuidad en los seres humanos.

Los autores consideran que en el caso de las hormonas endógenas humanas se debe determinar el porcentaje de aumento sobre los niveles basales al consumir carnes que las contengan, que si bien, los expertos de la OMS plantean como valores muy bajos con relación a la producción diaria humana, podrían generar efectos fisiológicos no deseados por la misma hormona, y causar alteraciones biológicas aún no establecidos, los cuales al aumentar una cantidad adicional a la producida por el cuerpo de manera natural, se pueden alterar los efectos fisiológicos generados por la misma hormona, y crear cambios importantes aún no establecidos.

Se requiere reevaluar los límites de seguridad y establecer los riesgos derivados de la ingesta de carnes y otros tejidos de animales tratados con hormonas esteroideas y otras sustancias, especialmente en niños, mujeres embarazadas y ancianos cuya susceptibilidad al efecto hormonal podría ser mayor.

Es necesario que las entidades responsables de vigilar la salud pública en el país determinen frecuentemente los niveles de sustancias permitidas como promotores de crecimiento y ajusten las recomendaciones a los niveles máximos establecidos por el Codex Alimentarius. Adicionalmente, se debe buscar en carnes de consumo humano sustancias no permitidas por la legislación colombiana.

Colombia debe definir las sustancias que pueden ser usadas y los límites permitidos, y a futuro, su eliminación completa para la producción animal. Es necesario establecer la resolución de prohibición del dietilestilbestrol.

Se deben establecer estudios orientados a determinar los efectos de las sustancias utilizadas en la producción animal, mediante, por ejemplo: HPLC, muestras de tejidos, usando especies menores u otras metodologías enunciadas dentro del presente artículo. Así mismo, se debe indagar sobre cuál es la relación que existe entre la dosis administrada del compuesto al animal y la dosis que puede encontrarse como residuo en la carne después de su sacrificio.

La eliminación del uso de antibióticos como promotores del crecimiento, debería ser el camino a seguir, pero se deben buscar nuevas alternativas a la producción animal como el uso de probióticos, prebióticos y sinergistas, que no dejan residuos en las carnes de consumo y por supuesto no afectarían la salud humana. 
Los autores consideran que la legislación colombiana debe establecer dentro de la normatividad para los mataderos, leyes que obliguen a desechar las orejas y la papada de los animales sacrificados, mientras se permita el uso de sustancias como promotores de crecimiento.

Dentro de la normatividad colombiana se encuentran contemplados aspectos como la manipulación de ejemplares desde el momento del nacimiento hasta el sacrificio y su posterior comercialización. Sin embargo, la falta de vigilancia y control deja abierta la posibilidad, de que el uso de sustancias permitidas se encuentren fuera de los límites mínimos recomendados por el Codex Alimentarius y además se usen sustancias prohibidas.

\section{Financiación}

Este trabajo fue financiado por los autores y parcialmente con recursos de la Fundación Universitaria del Área Andina.

\section{Conflicto de intereses}

Los autores expresan que no existe conflicto de intereses.

\section{Referencias}

1. Lozano M, Arias D. Residuos de fármacos en alimentos de origen animal: panorama actual en Colombia. Revista Colombiana de Ciencias Pecuarias. 2008; 21 (1): 23-34.

2. Martínez MJ. Repercusiones del implante de anabolizantes androgénicos sobre los perfiles hormonales de ganado vacuno. Tesis doctoral. Departamento de Fisiología Animal. Facultad de Veterinaria. Universidad Complutense de Madrid. 1993; 241 p.

3. Universidad de San Carlos de Guatemala. Centro Universitario de Sur Occidente Dirección general de investigación. Instituto de investigación de sur occidente -IIDESO- Determinación de esteroides anabólicos en carne de ganado bovino proveniente de la región sur occidental de Guatemala”. Mazatenango, noviembre de 2008. Biblioteca Virtual DIGI. http://digi.usac.edu.gt/bvirtual

4. www.produccion-animal.com.ar. Consultado 9 agosto 2010 .

5. Larrea-F, Chirinos-M. Impact on human health of hormonal additives used in animal production. Revista Investigaciones Clínicas. 2007; 59 (3): 206-211.

6. Torres-Zarazaga C. Antibióticos como promotores del crecimiento en animales. ¿Vamos por el buen camino? Gaceta Sanitaria, 2002; 16 (2).
7. Castro-M, Rodríguez-F. Levaduras: probióticos y prebióticos que mejoran la producción animal. Revista Corpoica, 2005; 6 (1).

8. Kabir-J, Umoh V, Audu-okoh E, Umoh J, Kwaga J. Veterinary drug use in poultry farms and determination of antimicrobial drug residues in commercial eggs and slaughtered chicken in Kaduna State, Nigeria. Food Control. 2004; 15 (1): 99-105.

9. Joint FAO/WHO Expert Committee on Food Additives. Residues of some veterinary drugs in animals and foods. FAO Food Nutrition Paper. 1988; 41 (1): 1-49.

10. JECFA Joint FAO/WHO Expert Committee on Food Additives, Evaluation of certain veterinary drug residues in food, WHO Technical Report Series. 2000; 893 (1): 21-54.

11. JECFA. Joint FAO/WHO Expert Committee on Food Additives, Evaluation of certain veterinary drug residues in food, WHO Technical Report Series. 1999; 888 (1): 12-45.

12. Mitchell-G A, Dunnavan-G. Illegal Use of b-Adrenergic Agonists in the United States, Journal Animal Scientific. 1998; 76 (1): 208-211.

13. Pulce-C, Lamaison-D, Keck-G, Bostvironnois-C, Nicolas J. Collective human food poisonings by clenbuterol residues in veal liver, Veterinary Human Toxicology. 1991; 33: 480-481.

14. Doyle-ME. Veterinary drug residues in processed meats - potential health risk. Food Research Institute, 2006 URL: http://www.wisc.edu/fri/briefs/FRIBrief_Vet DrgRes.pdf Consultado 8 de mayo de 2010

15. Farnot-Cardozo U. ¿Podemos aprender de los errores del pasado? Revista Cubana Salud Pública. 2007; 33 (4): 2-23.

16. Herbs AL. DES Associated clear cell adenocarcinoma of the vagina and cervix. Obstetric Gynecology Survival. 979; 34 (11): 844.

17. Herbst-AL, Scully-RE, Robboy-SJ. Prenatal diethylstilbestrol exposure and human genital tract abnormalities, National Cancer Institute monograph. 1979; (51): 25-35.

18. Gerbie-MV. Management of the adolescent girl exposed in utero to DES. Pediatry Annual. 1981; 10 (2): 23-26.

19. OMS. Boletín farmacéutico No 4. 2004; 23-87.

20. JECFA Joint FAO/WHO Expert Committee on Food Additives. Evaluation of certain veterinary drug residues in food, WHO Technical Report Series. 1990; 799: 12-65.

21. Paige-JC, Tollefson-L, Miller-MA. Health implications of residues of veterinary drugs and chemicals in 
animal tissues. Veterinary Clinical North American Food Animal Practice. 1999; 15 (1): 31-43.

22. Stachel-CS, Bock-C, Hamann-F, Gowik-P. Residues of several nitrofurans in egg. Journal Veterinary Pharmacology Therapy. 2006; 29: 120-143.

23. FAO/OMS, Residuos de medicamentos veterinarios en los alimentos. 32a Reunión de la Comisión del Codex Alimentarius 2009; 78. Disponible en: http:// www.codexalimentarius.net/vetdrugs/data/vetdrugs/ classes.html. Consultado 10 abril 2010.

24. Gardner D, Shobaack D. Endocrinología básica y clínica de Greenspan. Editorial Manual moderno. Séptima edición. ISBN-13:978-970-729-305-2. México. 2008; 993-1010.

25. CEE. Directiva 2003/74/CE del parlamento europeo y del consejo del 22 de septiembre de 2003; 45.

26. CEE. Directiva 96/22/CE del parlamento europeo y del consejo. Disposiciones 4,5,7. 1996; 56.

27. Comité Nacional del Codex Alimentarius. Colombia. 1998; 23-90.

28. ICA Instituto Colombiano Agropecuario. Resolución Nº 991 de 2004. Bogotá: ICA.

29. ICA Instituto Colombiano Agropecuario. Resolución N 1966 de 1984. Bogotá: ICA.

30. ICA Instituto Colombiano Agropecuario. Resolución Nº961 de 2003. Bogotá: ICA.

31. ICA Instituto Colombiano Agropecuario. Resolución N 1082 de 1995. Bogotá: ICA.

32. ICA Instituto Colombiano Agropecuario. Resolución № 1326 de 1981. Bogotá: ICA.

33. Organización de las Naciones Unidas para la agricultura y la alimentación. ALINORM 06/29/42 parte 196. Compendio de métodos de análisis identificados como idóneos para respaldar los LMR del Codex elaborado por el comité del Codex sobre residuos de medicamentos veterinarios en los alimentos. Disponible en: http://www.codexalimentarius.net/vetdrugs/ data/index.html?lang=es. Consultado 8 julio 2010.

34. Chrusch J, Lee S, Fedeniuk-R, Boison-JO. Determination of the performance characteristics of a new multiresidue method for non-steroidal anti-inflammatory drugs, corticosteroids and anabolic steroids in food animal tissues. Food additives \& contaminants Part A Chemical Analysis, control, exposure \& risk assessment. 2008; 25 (12): 1482-1496.

35. Xu-C L, Chu-X G, Peng-C F, Jin-Z Y, Wang-L Y. Development of a faster determination of 10 anabolic steroids residues in animal muscle tissues by liquid chromatography tandem mass spectrometry.Journal of pharmaceutical and biomedical analysis 2006; 41 (2): 616-621.

36. Blasco C, Van-Poucke C, Van-Peteghem C. Analysis of meat samples for anabolic steroids residues by liquid chromatography/tandem mass spectrometry. Journal Chromatography Annual. 2007; 1154 (1-2): 230-239.

37. Destrez B, Bichon E, Rambaud L, Courant F, Monteau F, Pinel G, Antignac JP, Le Bizec B. Criteria to distinguish between natural situations and illegal use of boldenone, boldenone esters and boldione in cattle 2. Direct measurement of 17 beta-boldenone sulphoconjugate in calf urine by liquid chromatographyhigh resolution and tandem mass spectrometry. Steroids. 2009; 74 (10-11): 803-808.

38. Furusawa N. A harmless method for determining trenbolone acetate together with 17 beta-trenbolone in beef. Journal of Chromatography. 2009; 47 (3): 243-246.

39. Duffy E, Rambaud L, Le Bizec B, O’Keeffe M. Determination of hormonal growth promoters in bovine hair: comparison of liquid chromatographymass spectrometry and gas chromatography-mass spectrometry methods for estradiol benzoate and nortestosterone decanoate. Analityca chimica Acta. 2009; 637 (1-2): 165-172.

40. Connolly L, Cai K, Van der Heiden E, Scippo ML, Muller M, Tarbin J, Elliott C. Detection of glucocorticoid bioactivity in bovine urine samples using a reporter gene assay. Analityca chimica Acta. 2009; 637 (1-2): 321-327.

41. Vincenti M, Girolami F, Capra P, Pazzi M, Carletti M, Gardini G, Nebbia C. Study of dexamethasone urinary excretion profile in cattle by LC-MS/MS: comparison between therapeutic and growth-promoting administration. Journal Agricultural and food chemistry 2009; 57 (4): 1299-1306.

42. Vascellari M, Pozza G, Poppi L, Capello K, Angeletti R, Ravarotto L, Andrighetto I, Mutinelli F. Evaluation of indirect biomarkers for detecting corticosteroids used as illegal growth promoters in beef cattle. Veterinary Rec. 2008; 163 (5): 147-151.

43. Sawaya WN, Lone K, Saeed T, Husain A, Khalafawi S. Application of an enzyme-linked immunosorbent assay for screening of sheep urine and animal tissue for the androgenic steroid trenbolone acetate in the State of Kuwait. Food additives \& Contaminants. 1998; 15 (2): 151-156.

44. Mattar S, Calderón A, Sotelo D, Sierra M, Tordecilla, G. Detección de antibióticos en leches: un problema de salud pública. Revista Salud Pública. 2009; 11 (4): 579-590.

45. Franco J, Romero M, Taborda G. Determinación de niveles residuales de tetraciclina en canales bovinos por la técnica de Elisa. Biosalud; 2008; 7: 47-55.

46. Marín-Lasso S, García-Matiz L. Niveles sanguíneos de ampicilina en pollos de engorde. Revista ICA, 1990; 25 (2): 87-93. 